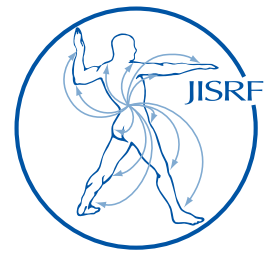

Check for updates

\title{
A Minimum 2-Year Follow-up Using Modular Trabecular Metal Tibial Components in Total Knee Arthroplasty
}

Zandee van Rilland $E^{1}$, Varcadipane $\mathrm{J}^{2}$, Geling $\mathrm{O}^{3}$, Murai Kuba $\mathrm{M}^{2}$, Nakasone $\mathrm{C}^{2}$

\begin{abstract}
Introduction: Early failure of tibial components remains a concern in total knee arthroplasty (TKA). Loss of fixation with cemented implants continues to be problematic in young, active patients. We sought to determine outcomes in patients receiving trabecular metal (TM) implants in a single-surgeon community hospital setting.

Methods: A retrospective analysis was performed on 167 consecutive primary TKAs performed on 133 patients utilizing a TM tibial implant with a minimum two years follow-up.

Results: Failure due to aseptic loosening occurred in 4 of the 167 cases (2.4\%). Local and systemic complication rates were low. Length of hospital stay and tourniquet time data were also reported.

Conclusion: Overall complications were low in our cohort of patients receiving TM implants. Longer follow-up is necessary to determine if the outcomes we observed are sustained over time.
\end{abstract}

Keywords: total knee arthroplasty; uncemented; cementless; trabecular metal; porous tantalum Level of Evidence: AAOS Therapeutic Level IV

\section{Introduction}

Among the many ways by which total knee arthroplasty (TKA) fail and require revision, early aseptic failure of tibial implants continues to be a major concern for most surgeons $[1,2,3]$. In the cemented TKA literature, aseptic loosening rates continue to remain between 1 - $7 \%$ in most reported series $[4,5,6,7,8]$. Although cemented TKA remains the gold standard, the bone cement interface represents a biologically static construct. Mechanical forces that overwhelm the strength of this interface will lead to failure without the biologic capacity for repair [9]. Biologic fixation of an implant to bone, if achieved, has the theoretical
1 University of Maryland School of Medicine, $620 \mathrm{~W}$ Lexington St, Baltimore, MD 21201 US

2 Straub Clinic Bone and Joint Center, 888 S. King St., Honolulu, HI 96813 US (Direct reprint requests to Cass Nakasone)

3 University of Hawaii at Manoa, 2500 Campus Rd, Honolulu, HI 96822 US

(C) 2015 Zandee van Rilland, Varcadipane, Geling, Murai Kuba, Nakasone. All rights reserved

Authors retain copyright and grant the journal right of first publication with the work. Reconstructive Review follows the Creative Commons Attribution-NonCommercial CC BY-NC. This license allows anyone to download works, build upon the material, and share them with others for non-commercial purposes as long as they credit the senior author, Reconstructive Review, and the Joint Implant Surgery \& Research Foundation (JISRF). An example credit would be: "Courtesy of (senior author's name), Reconstructive Review, JISRF, Chagrin Falls, Ohio". 
advantage of biologic repair throughout the life of the implant if the surrounding host bone maintains the ability for fracture repair and remodeling. Cementless implants were developed to capitalize on this potential $[10,11,12,13]$.

Early cementless porous-coated implants had several design flaws such as low porosity, suboptimal frictional characteristics, and increased modulus of elasticity as compared to bone [14]. Trabecular metal (TM) tibial components (Zimmer Inc.) were introduced in 1999 with the aim of overcoming the limitations of earlier porous coated cementless designs. The TM material is composed of tantalum, a chemically stable and inert metal with a high fatigue strength that makes it ideal for orthopaedic implants [14]. During development, the polyethylene tray is firmly attached to the TM material through direct compression molding. The TM tibial baseplate is additionally composed of two hexagon-shaped tantalum pegs that are press fit into holes drilled in the tibia to maximize bony ingrowth potential. Trabecular metal has demonstrated significant bony ingrowth and has been successfully used previously in tumor reconstruction, spinal fusion, and treatment of avascular necrosis of the femoral head [14]. With a porosity of up to $80 \%$ and a high coefficient of friction against cancellous bone, TM has great potential for biologic fixation [15]. The high porosity provides the ideal environment for bony in-growth and vascularization [16].

The advent of TM and early studies demonstrating better initial stabilization and comparable survival rates when compared to cemented tibial implants has led to a gradual increase in clinical use. The purpose of this study was to report our experiences using TM tibial components in a high volume, single-surgeon community hospital practice with a minimum of two years follow-up.

\section{Methods}

The study used prospectively collected data on all patients undergoing TKA with TM tibial baseplates performed by a single fellowship-trained orthopedic surgeon at a large community hospital. The study included all primary TKAs using TM tibial implants performed between October 2008 and May 2011, for a total of 167 TKAs performed on 133 patients. All patients consented for the procedure.

The initial Institutional Review Board approval for the study and data collection was obtained in 2004 and has been renewed annually. Data were collected prospectively using standardized data collection forms and protocols, and maintained in a designated database. Data were collected preoperatively and postoperatively, with the data- base continually updated with complications and followup data on all patients within the database. Standardized data collection forms were used to collect data on patient characteristics. The study inclusion criteria included any patient seen by the surgeon during the study time period for primary TKA with a TM tibial implant, including patients requiring hardware removal prior to primary arthroplasty. Patients undergoing revision TKA were excluded from this study.

The senior author $(\mathrm{CKN})$ performed all operations. All tibial implants were posterior stabilized modular components. Monoblock components were not used during this study. All trabecular metal tibial implants were by Zimmer (NexGen Trabecular Metal Tibial Tray).

All TKAs in this study were performed in a large community hospital and the study was observational in nature. The decision to use the modular TM tibial baseplate was made by the surgeon based on intraoperative assessment of bone quality. As there are no established quantitative measures or guidelines for surgeons to follow regarding the indications or contraindications for the use of trabecular metal implants, the surgeon (CKN) based his decision regarding the appropriateness of TM use on the visual inspection of bone porosity, age of the patient, bony defects present, and palpable assessment of bone density. The patella was routinely resurfaced and cemented. All femoral components were cemented.

All patients received the same preoperative and postoperative protocols. The schedule for follow-up consisted of a standard total knee postoperative protocol, which consisted of scheduled appointments at 2 weeks, 6 weeks, 3 months, 6 months, 1 year, and 2 years following surgery. Radiograph images were taken at each follow-up appointment to monitor implant fixation (Figure 1a-1c) and revision TKA was performed when necessary (Figure 1d). Beyond 2 years post-TKA, follow-up appointments were scheduled at 5 years or at the patient's preference.

All patients had hip-to-ankle radiographs prior to surgery and at 6-weeks follow-up to assess preoperative deformity and final outcome regarding implant position and mechanical alignment. All patients also had standard anteroposterior (AP), lateral, and merchant views done preoperatively and at each follow-up appointment. All patients had standard AP and lateral immediate postoperative radiographs taken in the recovery room following surgery.

Intraoperative complications, perioperative complications, and complications which occurred throughout the entire clinical follow-up period were prospectively collected and entered into a database. All pertinent surgical information including anesthesia type, tourniquet times, implant sizes and brand as well as patellar thickness pre- and 

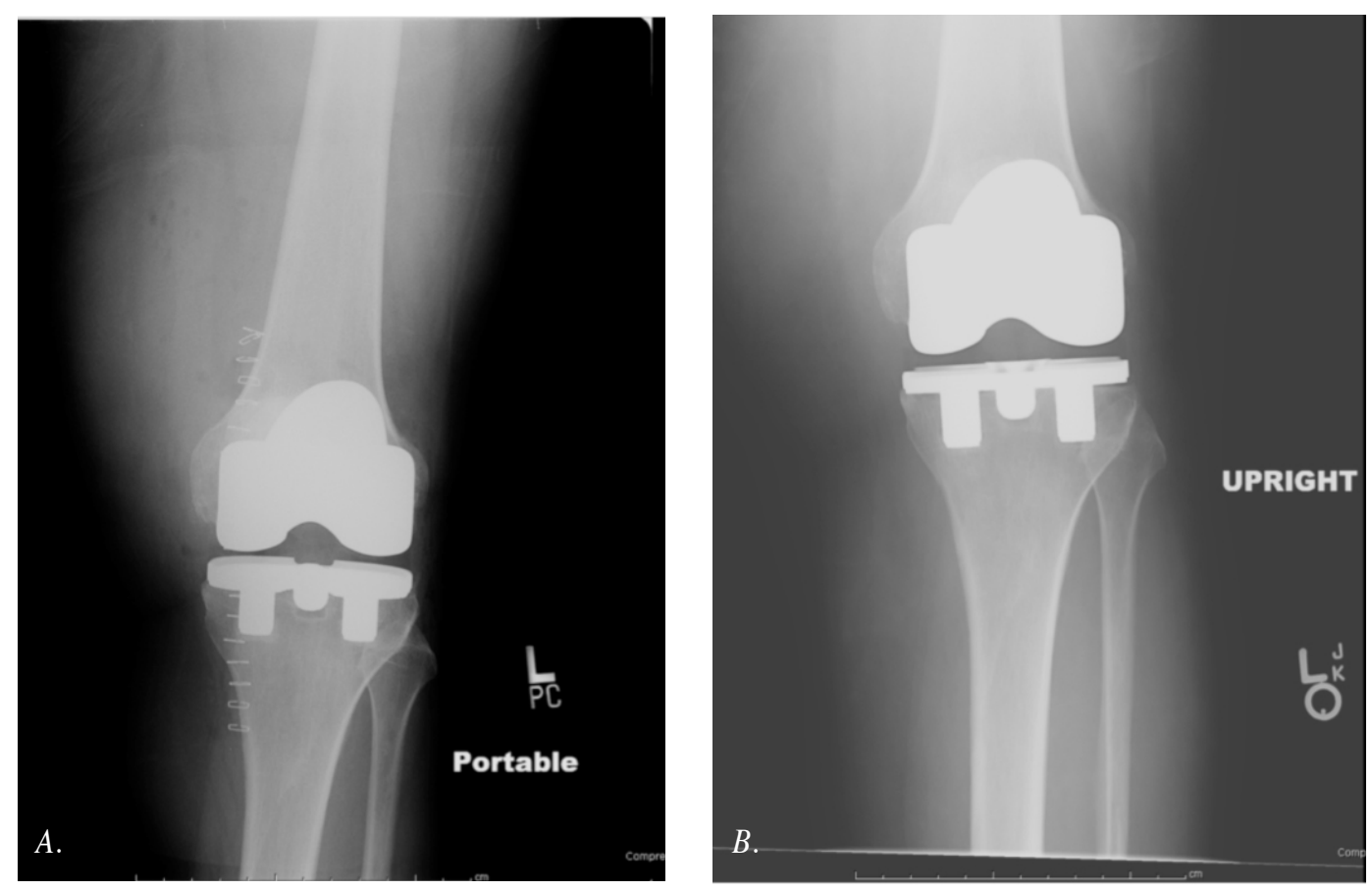

Figure 1. Radiograph images of TKA with TM tibial implants. Images taken (a) prior to discharge, (b) at 6 weeks follow-up, (c) at 8 months follow-up, and (d) following revision TKA.
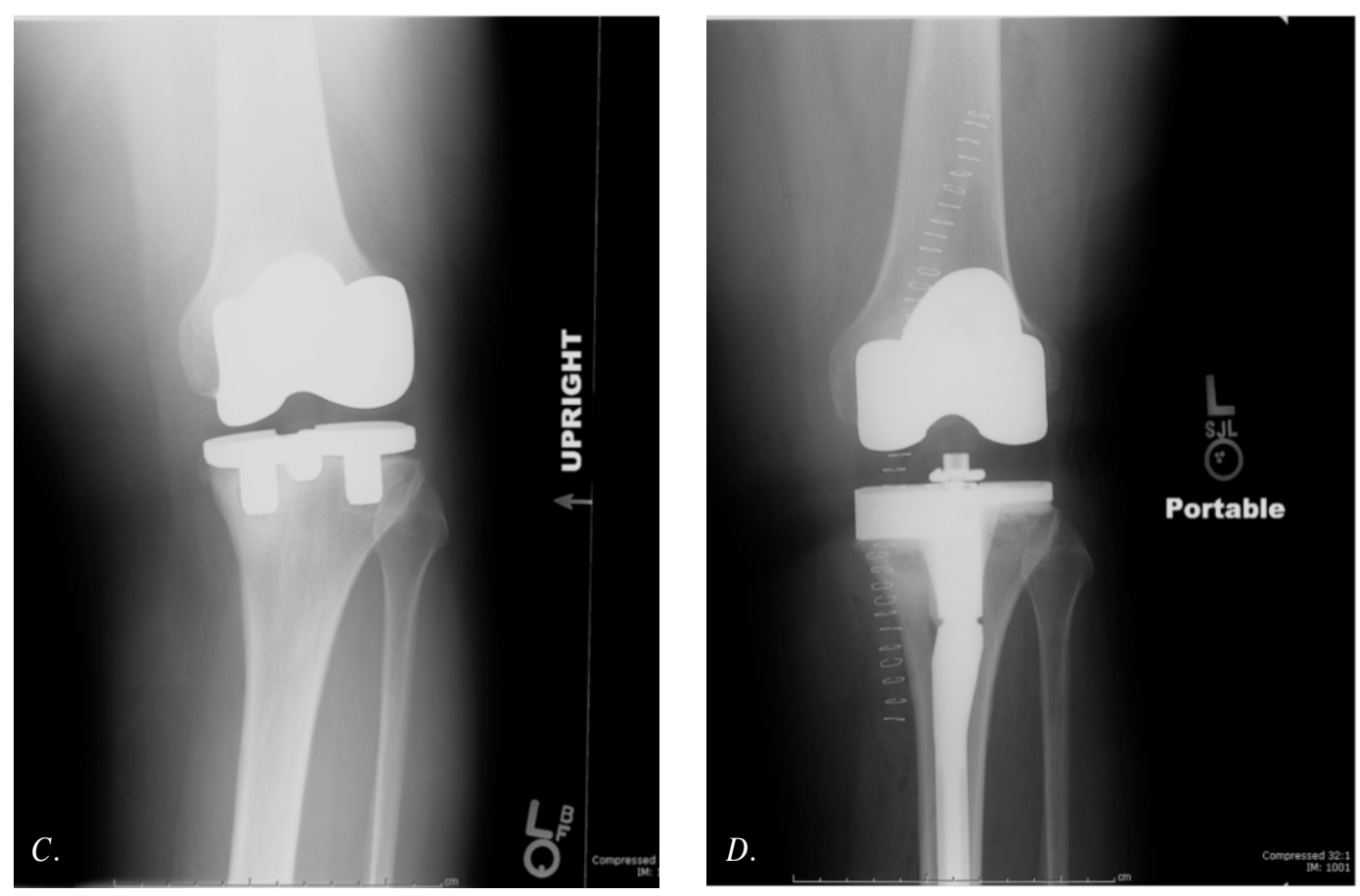

post-resurfacing were entered into the database. Preoperative knee range of motion, as well as immediate postoperative range of motion following wound closure and dressing application, was also recorded.

Aseptic failure was recorded per knee and was defined by either a grossly unstable radiograph or a bone scan positive for increased activity at the bone-implant interface with accompanying clinical symptoms. Clinical symptoms, which included the presence of progressive pain, chronic pain, or start-up pain, were investigated with laboratory tests to rule out infection including $\mathrm{C}$-reactive pro- tein, sedimentation rates, and aspirations where clinically indicated. Radiographic evidence of failure included gross subsidence of an implant, a lack of bony integration as determined by the lack of spot welding, or the presence of radiolucent lines or areas of osteolysis beneath the tibial baseplate. The onset of failure was defined as the day of diagnosis by the operating surgeon. 


\section{Results}

\section{Patient Characteristics}

Table 1 reports patient demographics and average follow-up time. Sex was reported as a percentage of males receiving TM implants. Mean age, body mass index (BMI), American Society of Anesthesiologists (ASA) Physical Status, and follow-up were also recorded.

\section{Local Complications}

Table 2 presents data on failure due to aseptic loosening and additional local complications recorded per knee $(n=167)$. These include deep venous thrombosis (DVT), peroneal nerve palsy, deep infection, superficial infection, wound dehiscence, structural implant failure, and fracture. Overall, the incidence of local complications was low.

\section{Systemic Complications}

Table 3 presents data on systemic complications recorded per patient $(n=133)$. Systemic complications included myocardial infarction (MI), pulmonary embolism (PE), and death. Overall, incidence rates of systemic complications were low.

\section{Tourniquet Time and Length of Stay}

Tourniquet time was analyzed and recorded "per TKA" whereas length of stay (LOS) was analyzed and recorded "per surgery". The average tourniquet time was $47.6 \mathrm{~min}$ utes (range 0-90 minutes). No tourniquet was used in two knees undergoing TKA with a TM tibial component.

The LOS (per surgery) was 2.81 days $(\mathrm{SD}=1.3$ days, range 1-9 days) with the median length being 2.0 days.

\section{Implant Survival}

The failure time was defined as the time between the date of the TKA and the earliest clinical or radiographic indication of aseptic failure. All 4 cases (100\%) of aseptic failure occurred within 1 year (two failures at 1 month, one case at 2 months, and one case at 8 months). The mean time to aseptic failure following TKA was 3.5 months (range: 1-8 months).

\section{X-Ray Review Analysis}

While we understand that the KSS and radiographic data is valuable in demonstrating clinical efficacy, we do not have a completed data set to perform the analysis to include in this paper.
Table 1: Patient Characteristics

\begin{tabular}{|l|l|}
\hline Variable & TM Baseplate $(\mathbf{n = 1 3 3 )}$ \\
\hline Male & $83(62.4 \%)$ \\
\hline Age (years) & $65.5 \pm 8.9$ \\
\hline BMI & $31.9 \pm 5.4$ \\
\hline ASA Physical Status & $2.2 \pm 0.5$ \\
\hline Follow-up (months) & $33.9 \pm 8.4$ \\
\hline
\end{tabular}

Note: Data are presented per patient. The values presented for age, BMI, ASA, and follow-up are the means and standard deviations.

Table 2: Local Complications

\begin{tabular}{|l|l|}
\hline Complication & TM Base plate $(\mathbf{n = 1 6 7})$ \\
\hline Aseptic Loosening & $4(2.4 \%)$ \\
\hline DVT & $4(2.4 \%)$ \\
\hline Peroneal Nerve Palsy & 0 \\
\hline Deep Infection & $4(2.4 \%)$ \\
\hline Superficial Infection & 0 \\
\hline Wound Dehiscence & 0 \\
\hline Structural Implant Failure & 0 \\
\hline Fracture & 0 \\
\hline TOTAL & $12(7.2 \%)$ \\
\hline
\end{tabular}

Note: Data are presented per TKA.

Table 3: Systemic Complications

\begin{tabular}{|l|l|}
\hline Complication & TM Base plate $(\mathbf{n}=\mathbf{1 3 3})$ \\
\hline Death & 0 \\
\hline MI & 0 \\
\hline PE & $1(.8 \%)$ \\
\hline TOTAL & $1(.8 \%)$ \\
\hline
\end{tabular}

Note: Data are presented per patient.

\section{Discussion}

This study reports our experiences with TKA in patients receiving cementless TM tibial components using prospectively collected data on 167 TKAs performed on 133 patients. Data was collected in a large volume, single-surgeon practice over a three year period with a minimum two years of follow-up. In our series, the early aseptic failure rate in patients undergoing TKA with TM tibial components was $2.4 \%$.

Despite the biologic promise of trabecular metal, there is relatively little reported experience utilizing TM tibial implants compared to the overwhelmingly large data available regarding cemented TKA. Initial research by Bobyn et al demonstrated low complication rates yet had a limit- 
ed number of patients and an undefined follow-up period [17]. Helm et al followed 105 patients for three years and found no radiolucencies, with only one revision of the tibial component (1\%), which was attributed to a trauma [18]. Ghalayini et al reported similar results at 6 years [19]. Unger et al followed 108 patients for two years and had no revisions or clinical or radiographic failures [20]. Kamath et al reported on 100 consecutive patients younger than 55 at a minimum of 5 years and found no significant difference to a matched cemented group and noted no failures due to fixation [21].

Aseptic loosening of the tibial component remains a primary cause of revision surgery in TKA. Multiple studies have reported aseptic loosening to account for between $3 \%$ and $60 \%$ of TKA failures depending on the length of follow-up [22,23,24,25,26,27]. Research comparing aseptic failure rates in cementless TM tibial components to cemented tibial components is limited. A study by Dunbar et al [2] used radiostereometric analysis to evaluate migration of the tibial components, and found the proportion of components "at risk" of aseptic loosening to be $0 \%$ in patients with a TM tibial component and $19 \%$ in patients with cemented tibial components at 2 years follow-up. A similar study at 5 years follow-up demonstrated solid fixation and stabilization of the TM tibial components with $0 \%$ of TM components at risk of aseptic loosening [28]. Hayakawa et al reported no cases of implant loosening in their series of 29 TM tibial component TKA patients [29]. Fernandez-Fairen et al similarly reported no cases of aseptic loosening in any of their $69 \mathrm{TM}$ tibial component and 63 cemented tibial component patients [30]. A recent randomized control trial comparing cemented and TM tibial component survivorship found that patients receiving TM tibial implants had a lower 5-year risk of aseptic loosening than patients receiving cemented tibial implants [31]. Survivorship and functionality were comparable between the two groups.

In our study, there were four cases $(2.4 \%)$ of aseptic loosening in patients receiving TM tibial components. In addition, all of our patients were seen at a minimum of two years following surgery during which time the majority of failures would be expected [2,32]. Indeed all four cases of aseptic loosening occurred within 8 months. Two cases of aseptic failure occurred prior to 1-month follow-up. Both cases had varus subsidence of the tibial plate indicating aseptic loosening as the primary mechanism of failure.

The mechanism by which aseptic failures occur in TM tibial implants may also be postulated by observations in our study. Earlier reports demonstrated that the majority of aseptic failures cases occurred within the first two years following TKA [32]. We report similar results in our series, as all 4 cases of aseptic failure occurred within 8 months following surgery. This finding may shed light on the mechanism by which aseptic loosening occurs in TM TKA. Trabecular metal implants allow for dynamic bone remodeling at the bone-implant interface if biologic fixation succeeds. Aseptic failure with TM implants would be expected early if osseointegration fails, which was observed in our study. On the other hand, cemented implants form a static interface with bone, which can gradually break down and loosen over time, resulting in a greater incidence of aseptic loosening over time. More prospective, randomized studies looking at the survival of these implants over the long-term should be done to evaluate their longevity.

Tourniquet time can be used as a way to measure surgery time, as well as a means to quantify efficiency. Since TM tibial components do not require cement, time spent on preparing the cement can be saved. Furthermore, there are disadvantages to prolonged operative and tourniquet times in addition to the added operating room costs. These include increased blood loss, tourniquet associated ischemia, increased incidence of infection, and delayed wound healing [33]. However, the cost benefit gained in the shorter operating time may be slightly offset by the higher cost of the TM tibial component.

There have been reported drawbacks to the use of TM in TKA. In a case report of an 80 year-old man receiving a TM tibial component, it was hypothesized that a patient's advanced age may cause reduced regenerative ability of bone, and thus diminished effectiveness of the TM tibial component [34]. This hypothesis raises the important question: how do orthopedic surgeons determine who is an appropriate candidate for a TM implant? We may never be able to identify specific characteristics that give us reliable answers, but this limited study appears to support the use of TM baseplates in younger male patients.

The primary potential study limitation was the observational nature of the study. The surgeon used his clinical judgment to determine if a patient was suitable to receive a TM tibial implant. As a result, the patients in this series were relatively young (65.5 years), and predominantly male (62.4\%). However, previous studies have demonstrated higher TKA failure rates in younger patients [35] and in males [36,37]. Based on the findings of these previous studies, as well as the low aseptic failure rate in our series of patients, one could postulate that TM implants may be an effective alternative for younger, active patients. Randomized trials with long-term follow-ups comparing TM to cemented tibial implants are necessary to determine the clinical worth of TM. 


\section{Conclusion}

The demand for knee replacements is expected to continue to rise, particularly in younger patient populations $[38,39]$. Biologic fixation of implants has theoretical advantages over static fixation strategies such as cement. We report an aseptic loosening rate of $2.4 \%$ in our cohort of TKA patients receiving TM implants. Additional local and systemic complications were rare. A substantial amount of research is still required to determine if TM implants will provide improved longevity in TKA patients.

\section{References}

1. Bourne RB, Finlay JB. The influence of tibial component intramedullary stems and implant-cortex contact on the strain distribution of the proximal tibia following total knee arthroplasty: an in vitro study. Clin Orthop Relat Res. 1986;208:9599

2. Dunbar MJ, Wilson DAJ, Hennigar AW, Amirault JD, Gross M, Reardon GP. Fixation of a trabecular metal knee arthroplasty component. A prospective randomized study. J Bone Joint Surg Am. 2009;91:1578-1586

3. Cawley DT, Kelly N, Simpkin A, Shannon FJ, McGarry JP. Full and surface tibial cementation in total knee arthroplasty: a biomechanical investigation of stress distribution and remodeling in the tibia. Clin Biomech (Bristol, Avon). 2012;27:390397

4. Foran JR, Whited BW, Sporer SM. Early aseptic loosening with a precoated lowprofile tibial component: a case series. J Arthroplasty. 2011;26:1445-1450

5. Barrington JW, Sah A, Malchau H, Burke DW. Contemporary cruciate-retaining total knee arthroplasty with a pegged tibial baseplate. Results at a minimum of ten years. J Bone Joint Surg Am. 2009;91:874-878

6. Miller CW, Pettygrow R. Long-term clinical and radiographic results of a pegged tibial baseplate in primary total knee arthroplasty. J Arthroplasty 2001;16:70

7. Weeden SH, Ogden SB. Early loosening of MIS tibial implants in primary TKA. American Academy of Orthopaedic Surgeons Annual Meeting. New Orleans, LA; 2010

8. Anderson DR, Anderson JT. High incidence of aseptic loosening following MIS total knee arthroplasty at short- term follow-up. American Academy of Orthopaedic Surgeons Annual Meeting. Las Vegas, NV; 2009

9. Lewis G. Properties of acrylic bone cement: state of the art review. J Biomed Mater Res. 1997;38:155-182

10. Soballe K, Hansen ES, Brockstedt-Rasmussen H, Bunger C. Hydroxyapatite coating converts fibrous tissue to bone around loaded implants. J Bone Joint Surg Br. 1993; $75: 270-278$

11. Hofmann AA, Evanich JD, Ferguson RP, Camargo MP. Ten- to 14-year clinical followup of the cementless Natural Knee system. Clin Orthop Relat Res. 2001;388:85-94

12. Baldwin JL, El-Saied MR, Rubinstein RA Jr. Uncemented total knee arthroplasty report of 109 titanium knees with cancellous-structured porous coating. Orthopedics. 1996;19:123-130

13. Hofmann AA, Heithoff SM, Camargo M. Cementless total knee arthroplasty in patients 50 years or younger. Clin Orthop Relat Res. 2002;404:102-107

14. Patil N, Lee K, Goodman SB. Porous tantalum in hip and knee reconstructive surgery. J Biomed Mater Res B Appl Biomater. 2009;89:242-251

15. Bobyn JD, Stackpool GJ, Hacking SA, Tanzer M, Krygier JJ. Characteristics of bone ingrowth and interface mechanics of a new porous tantalum biomaterial. J Bone Joint Surg Br. 1999;81:907-914

16. Karageorgiou V, Kaplan D. Porosity of 3D biomaterial scaffolds and osteogenesis. Biomaterials. 2005;26:5474-5491

17. Bobyn JD, Poggie RA, Krygier JJ, Lewallen DG, Hanssen AD, Lewis RJ, Unger AS, et al. Clinical validation of a structural porous tantalum biomaterial for adult reconstruction. J Bone Joint Surg Am. 2004;86A:123-129

18. Helm AT, Kerin C, Ghalayini SR, McLauchlan GJ. Preliminary results of an un- cemented trabecular metal tibial component in total knee arthroplasty. J Arthroplasty. 2009;24:941-944

19. Ghalayini SR, Helm AT, McLauchlan GJ. Minimum 6year results of an uncemented trabecular metal tibial component in total knee arthroplasty. The Knee. $2012 \cdot 19 \cdot 872-874$

20. Unger AS, Duggan JP. Midterm results of a porous tantalum monoblock tibia component. J Arthroplasty. 2011;26:855-860

21. Kamath AF, Lee GC, Sheth NP, Nelson CL, Garino JP, Israelite CL. Prospective results of uncemented tantalum monoblock tibia in total knee arthroplasty: minimum 5-year follow-up in patients younger than 55 years. J Arthroplasty. 2011;26:1390-1395

22. Fehring TK, Odum S, Griffin WL, Mason JB, Nadaud M. Early failures in total knee arthroplasty. Clin Orthop Relat Res. 2001;392:315-318

23. Bozic KJ, Kurtz SM, Lau E, Ong K, Chiu V, Vail TP, Rubash HE, Berry DJ. The epidemiology of revision total knee arthroplasty in the United States. Clin Orthop Relat Res. 2010;468:45-51

24. Sharkey PF, Hozack WJ, Rothman RH, Shastri S, Jacoby SM. Insall Award paper. Why are total knee arthroplasties failing today? Clin Orthop Relat Res. 2002; $404: 7-13$

25. Vessely MB, Whaley AL, Harmsen WS, Schleck CD, Berry DJ. The Chitranjan Ranawat Award: Long-term survivorship and failure modes of 1000 cemented condylar total knee arthroplasties. Clin Orthop Relat Res. 2006;452:28-34

26. Peters CL, Hennessey R, Barden RM, Galante JO, Rosenberg AG. Revision total knee arthroplasty with a cemented posterior-stabilized or constrained condylar prosthesis: a minimum 3-year and average 5-year follow-up study. J Arthroplasty. 1997;12:896-903

27. Garcia RM, Hardy BT, Kraay MJ, Goldberg VM. Revision total knee arthroplasty for aseptic and septic causes in patients with rheumatoid arthritis. Clin Orthop Relat Res. 2010;468:82-89

28. Wilson DA, Richardson G, Hennigar AW, Dunbar MJ. Continued stabilization of trabecular metal tibial monoblock total knee arthroplasty components at 5 years measured with radiostereometric analysis. Acta Orthop. 2012;83:36-40

29. Hayakawa K, Date H, Tsujimura S, Nojiri S, Yamada H, Nakagawa K. Mid-term results of total knee arthroplasty with a porour tantalum monoblock tibial component. Knee. 2014;21(1):199-203

30. Fernandez-Fiaren M, Hernandez-Vaquero D, Murcia A, Torres A, Llopis R. Trabecular metal in total knee arhtroplasty associated with higher knee scores: a randomized controlled trial. Clin Orthop Relat Res. 2013;471:3543-3553

31. Pulido L, Abdel MP, Lewallen DG, Stuart MJ, Sanchez-Sotelo J, Hanssen AD Pagnano MW. Trabecular Metal Tibial Components Were Durable and Reliable in Primary Total Knee Arthroplasty: A Randomized Clinical Trial. Clin Orthop Relat Res. 2014 Apr 1.

32. Piedade SR, Pinaroli A, Servien E, Neyret P. Revision after early aseptic failures in primary total knee arthroplasty. Knee Surg Sports Traumatol Arthrosc. 2009; $17: 248-253$

33. Cheng, T., Zhang, G., \& Zhang, X. (2011). Imageless Navigation System Does Not Improve Component Rotational Alignment in Total Knee Arthroplasty. Journal of Surgical Research, 171(2), 590-600.

34. Tigani D, Sabbioni G, Raimondi A. Early aseptic loosening of a porous tantalum knee prosthesis. Chir Organi Mov. 2009;93:187-191

35. Harrysson OL, Robertsson O, Nayfeh JF. Higher cumulative revision rate of knee arthroplasties in younger patients with osteoarthritis. Clin Orthop Relat Res 2004;421:162-168

36. Singh JA, Kwoh CK, Richardson D, Chen W, Ibrahim SA. Gender and surgica outcomes and mortality after primary total knee arthroplasty: A risk-adjusted analysis. Arthritis Care Res (Hoboken). 2013

37. Gioe TJ, Novak C, Sinner P, Ma W, Mehle S. Knee arthroplasty in the young patient: survival in a community registry. Clin Orthop Relat Res. 2007;464:83-87

38. Jain NB, Higgins LD, Ozumba D, Guller U, Cronin M, Pietrobon R, Katz JN Trends in epidemiology of knee arthroplasty in the United States, 1990-2000. Arthritis Rheum. 2005;52:3928-3933

39. Losina E, Thornhill TS, Rome BN, Wright J, Katz JN. The dramatic increase in total knee replacement utilization rates in the United States cannot be fully explained by growth in population size and the obesity epidemic. J Bone Joint Surg Am. 2012;94:201-20 